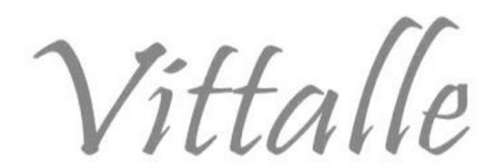

\title{
Emissões de gases de efeito estufa no estado de São Paulo: análise do setor de transportes e impactos na saúde
}

\author{
Vinicius Pazini Leite, Daniela Debone, Simone Georges El Khouri Miraglia* \\ Laboratório de Economia, Saúde e Poluição Ambiental da Universidade Federal de São Paulo (LESPA - UNIFESP), \\ Diadema, SP, Brasil
}

Histórico do Artigo:
Recebido em:
14/10/2020
Aceito em
02/11/2020

Palavras-chave: $\mathrm{CO}_{2}$; mudança climática; transportes; emissões de veículos; doenças respiratórias; doenças cardiovasculares

\section{Keywords:}

$\mathrm{CO}_{2}$; climate change; transportation; vehicle emissions; respiratory tract diseases; cardiovascular diseases

\begin{abstract}
RESUMO
As ações antrópicas têm sido responsáveis por incontáveis impactos negativos para a sociedade. Neste cenário, pode-se destacar as mudanças climáticas como um dos maiores desafios do século XXI, implicando em perdas socioeconômicas e à saúde global. Os grandes centros urbanos sofrem profundamente as consequências causadas pelas mudanças climáticas e degradação da qualidade do ar, bem como contribuem diretamente para a intensificação desses processos. $\mathrm{O}$ objetivo deste estudo foi avaliar qualitativamente as séries temporais de gases de efeito estufa e a mortalidade por causas cardiorrespiratórias do estado de São Paulo, Brasil, durante o período de 2000 a 2018. Nossos resultados demonstraram que o setor de transportes se mostrou o principal emissor de dióxido de carbono $\left(\mathrm{CO}_{2}\right)$ do estado, com média elevada de consumo de diesel, refletindo na forte correlação positiva e significativa entre as emissões e mortalidade por causas cardiovasculares e respiratórias, e assim, promovendo a importante discussão sobre a urgência de investimento em sistemas de transportes mais limpos, bem como soluções multimodais para o transporte de cargas e a necessidade de implementação de políticas adequadas de mitigação de emissões de $\mathrm{CO}_{2}$.
\end{abstract}

Greenhouse gas emissions in São paulo State: analysis of transport sector and health impacts

\section{ABSTRACT}

Anthropogenic actions have been responsible for countless negative impacts for contemporary society. In this scenario, climate change can be highlighted as one of the greatest challenges of the 21 st century, resulting in socioeconomic and global health losses. Large urban centers suffer deeply the consequences caused by climate change and air quality degradation, as well as directly contributing to the intensification of these processes. The objective of this study was to qualitatively evaluate the time series of greenhouse gases and mortality due to cardiorespiratory causes in the state of São Paulo, Brazil, during the period from 2000 to 2018. Our results showed that the transportation sector proved to be the main emitter of carbon dioxide $\left(\mathrm{CO}_{2}\right)$ in the state, with high average diesel consumption, reflecting the strong positive and significant correlation between these emissions and mortality from cardiovascular and respiratory causes, and thus, promoting the important discussion on the urgency of cleaner transport investment, as well as multimodal solutions for cargo transportation and the need to implement appropriate policies to mitigate $\mathrm{CO}_{2}$ emissions.

\section{Introdução}

Desde a segunda metade do século XVII, marcada pela Revolução Industrial, as ações antrópicas passaram a ser mais frequentes e intensas, em termos de exploração de serviços ecossistêmicos e recursos naturais, provocando incontáveis prejuízos para a sociedade e o meio ambiente. Dentre os impactos negativos gerados, pode-se destacar as mudanças climáticas, principal consequência da queima de combustíveis fósseis e emissões de gases de efeito estufa (GEE), como um dos maiores desafios do século XXI, implicando em perdas socioeconômicas e à saúde da população global (1-7).

\footnotetext{
*Autor correspondente: simone.miraglia@unifesp.br (Miraglia S.G.E.K)
} 
Os GEE são responsáveis por reter a energia emitida pelo sol, impedindo que parte dela seja refletida para o espaço, fazendo com que a temperatura média da Terra se mantenha próxima a $14^{\circ} \mathrm{C}$, na ausência deste fenômeno, a temperatura seria muito reduzida, o que impediria a existência de vida em nosso planeta (8). A queima de combustíveis fósseis como fonte de energia, mudança de uso da terra (desmatamento e queimadas) e agropecuária são os principais responsáveis pela emissão de bilhões de toneladas de GEE anualmente, intensificando este fenômeno e, portanto, provocando o aquecimento global e consequentes mudanças climáticas (9-11).

Dentre os principais gases de efeito estufa emitidos globalmente, destacam-se o dióxido de carbono $\left(\mathrm{CO}_{2}\right)$, o metano $\left(\mathrm{CH}_{4}\right)$ e o óxido nitroso $\left(\mathrm{N}_{2} \mathrm{O}\right)$. O $\mathrm{CO}_{2}$ representa cerca de $81 \%$ das emissões, é capaz de manter-se por centenas de anos na atmosfera e sua emissão é proveniente principalmente da queima de combustíveis fósseis (gasolina, diesel, gás natural e carvão mineral), queima de biomassa e processos industriais. $\mathrm{O}$ $\mathrm{CH}_{4}$ contribui com $10 \%$ do total emitido, é resultante principalmente da fermentação entérica de ruminantes, resíduos sólidos urbanos dispostos em aterros ou lixões e queima de biomassa. $\mathrm{O} \mathrm{N}_{2} \mathrm{O}$ contribui com cerca de $7 \%$ das emissões globais, é proveniente principalmente uso e produção de fertilizantes, manejo de dejetos animais provenientes da pecuária e transportes $(9,12)$.

A mudança do clima induzida pelo homem está intimamente associada ao aumento exagerado da temperatura global e da frequência de eventos extremos, tais como ondas de calor, incêndios, furacões e inundações (1-5). Projeções indicam, que na ausência de medidas mitigadoras, a temperatura média da superfície global poderá alcançar um aumento de 1,8 a $4,0{ }^{\circ} \mathrm{C}$ até o final do século (13). Entretanto, os efeitos negativos das temperaturas aumentadas já estão sendo sentidos ao redor do mundo. Nori-Sarma et al. (14) demonstraram que, na Índia, a mortalidade aumentou cerca de $18 \%$ em períodos que apresentaram ondas de calor, entre os anos de 2000 a 2012. Em 2010, eventos extremos de seca e ondas de calor resultaram para a Rússia cerca de 11.000 mortes e perda de $30 \%$ da colheita de trigo. No mesmo ano, o Paquistão vivenciou uma das piores enchentes de sua história, que alcançou uma extensão significativa de seu território, atingindo 20 milhões de pessoas (15). Guo et al. (16) destacaram que o Brasil se mostra muito vulnerável ao aumento da mortalidade de idosos com doenças cardiovasculares e respiratórias, durante ondas de calor. Além disso, estima-se que os custos anuais de mortes prematuras, devido aos níveis elevados de poluição atmosférica, sejam em torno de 1,7 bilhões de dólares, em 29 regiões metropolitanas brasileiras (17).

A queima de combustíveis fósseis não só contribui significativamente com o aumento das emissões $\mathrm{CO}_{2}$ (e dos demais gases de efeito estufa), mas também com o aumento da concentração de diversos poluentes atmosféricos locais, tais como material particulado (MP), monóxido de carbono $(\mathrm{CO})$, óxidos de nitrogênio $\left(\mathrm{NO}_{\mathrm{x}}\right)$, óxidos de enxofre $\left(\mathrm{SO}_{\mathrm{x}}\right)$ e hidrocarbonetos, que estão diretamente associados ao aumento do risco complicações cardiorrespiratórias (5,18-21). Assim, os grandes centros urbanos sofrem profundamente as consequências causadas pelas mudanças climáticas e a degradação da qualidade do ar, bem como contribuem diretamente para a intensificação desses processos $(3,4)$. Portanto, desempenham papel fundamental na promoção de estratégias e políticas públicas eficientes, voltadas à mitigação das emissões de gases de efeito estufa e ao controle da poluição atmosférica.

Neste sentido, vale destacar que as consequências das mudanças climáticas são sistêmicas e transfronteiriças, uma vez que podem afetar diretamente a saúde humana, a estabilidade socioeconômica e política, não se limita à faixa etária, classe social ou limite territorial específicos. Sabe-se que o alcance transfronteiriço da poluição atmosférica pode impactar significativamente a qualidade do ar e a saúde de uma região 
que recebeu poluentes de outro local. Por exemplo, o governo do Canadá relatou recentemente que mais de $50 \%$ da poluição do ar de Ontário tem origem norteamericana $(22,23)$. Semelhantemente, os impactos das mudanças climáticas afetam as desigualdades entre os países, uma vez que os países de baixa renda são e serão mais impactados com o aumento da temperatura global, eventos extremos e insegurança alimentar, bem como com os custos de mitigação. Portanto, a ação conjunta entre governos e sociedade é substancial para reverter este cenário de crise (4,24-26).

Localizado na região sudeste do Brasil, o estado de São Paulo possui 645 municípios, área territorial de $248.219 \mathrm{~km}^{2}$ e 46.289 .333 habitantes, de acordo com as estimativas mais atuais do Instituto Brasileiro de Geografia e Estatística (IBGE) (27). Trata-se do estado mais populoso do país e o maior em termos econômicos e industriais, contando com a maior frota veicular do Brasil, 30.574 .877 veículos motorizados, dos quais 693.391 são caminhões, de acordo com dados do Departamento Nacional de Trânsito (DENATRAN) de 2020 (28). Portanto, apresenta elevados índices de emissão de poluentes atmosféricos e gases de efeito estufa, bem como uma população altamente exposta aos riscos derivados das mudanças climáticas e degradação da qualidade do ar $(27,29,30)$. Entretanto, há poucos estudos publicados que investigaram a relação entre emissões de GEE e indicadores socioeconômicos para o estado.

Dessa maneira, o objetivo deste estudo foi avaliar a evolução temporal das emissões de GEE do estado de São Paulo, bem como a relação com os impactos na mortalidade cardiorrespiratória da população.

\section{Métodos}

$\mathrm{Na}$ presente pesquisa, foram utilizados dados secundários de fonte pública em escala anual, considerando, como período de análise, o intervalo entre os anos de 2000 e 2018. Os dados de emissões de gases de efeito estufa (GEE) foram coletados no Sistema de Estimativas de Emissões de Gases de Efeito Estufa (SEEG). Nas análises realizadas, foram considerados os principais gases de efeito estufa: $\mathrm{CO}_{2}, \mathrm{CH}_{4}$ e $\mathrm{N}_{2} \mathrm{O}$, com foco principal para emissões de $\mathrm{CO}_{2}$, principal GEE emitido no estado de São Paulo (31).

Os dados relativos ao consumo de combustível foram obtidos da Agência Nacional do Petróleo, Gás Natural e Biocombustíveis (ANP) (32). Nas análises realizadas, foram consideradas as médias de consumo de etanol, gasolina $\mathrm{C}$ e diesel, entre os anos de 2000 e 2018, e comparadas pelo teste estatístico ANOVA, seguido do pós-teste de Bonferroni.

Os óbitos totais decorrentes de doenças respiratórias e cardiovasculares foram obtidos do banco de dados do departamento de informática do Sistema Único de Saúde do Brasil (DATASUS) e as estimativas da população do estado foram obtidas do banco de dados da Fundação Sistema Estadual de Análise de Dados Estatísticos (Fundação SEADE) $(33,34)$. Assim, com essas variáveis, foram calculadas as taxas anuais de mortalidade, por causas respiratórias, cardiovasculares e cardiorrespiratórias (soma), ao longo da série temporal, de acordo com a Equação (1)

$$
\text { taxa de mortalidade }=\frac{\text { nůmero anual de óbitos }}{\text { estimativa anual da populaçầ }} \times 100.000
$$

Após verificação da distribuição normal das variáveis $\mathrm{CO}_{2}$ e mortalidade, a análise de correlação de Pearson foi realizada e o coeficiente de correlação foi calculado, de acordo com a Equação (2) 


$$
r=\frac{\sum_{i=1}^{n}\left(x_{i}-\bar{x}\right)\left(y_{i}-\bar{y}\right)}{\sqrt{\sum_{i=1}^{n}\left(x_{i}-\bar{x}\right)^{2} x \sum_{i=1}^{n}\left(y_{i}-\bar{y}\right)^{2}}}
$$

Para as análises estatísticas e produção de gráficos e tabelas, foram utilizados os softwares IBM SPSS versão 26.0, GraphPad Prism 5.0 e Microsoft Excel.

\section{Resultados e discussão}

A Figura 1 apresenta a série histórica dos principais GEE emitidos no estado de São Paulo. $\mathrm{O} \mathrm{CO}_{2}$ é emitido pela queima de combustíveis fósseis; a emissão de $\mathrm{CH}_{4}$ é representada principalmente pela fermentação entérica dos ruminantes e cultivo de arroz; e a emissão de $\mathrm{N}_{2} \mathrm{O}$ decorre principalmente da aplicação de fertilizantes nitrogenados e manejo de dejetos de animais $(9,35)$.

Pode-se observar que, ao longo de toda a série histórica analisada, o $\mathrm{CO}_{2}$ é principal GEE emitido, seguido do $\mathrm{CH}_{4}$ e do $\mathrm{N}_{2} \mathrm{O}$, com menor contribuição nas emissões estaduais totais. $\mathrm{O} \mathrm{CO}_{2}$ apresentou emissões sempre acima de 75 milhões de toneladas, com picos de emissões entre os anos de 2011 e 2015 e leve redução a partir de 2016, devido ao avanço da crise econômica (36). No geral, o $\mathrm{CH}_{4}$ apresentou leve tendência de aumento ao longo da série histórica, mantendo-se em uma faixa de 1,39 e 1,69 milhões de toneladas emitidas. $\mathrm{E}$ o $\mathrm{N}_{2} \mathrm{O}$, apresentou importante queda, principalmente após 2007, que pode ser explicada por processos regulatórios implementados para o setor sucroalcooleiro, com a Lei 11.241/2002, e também com a implementação do Protocolo Agroambiental, em 2007 (37). Assim, nos últimos 10 anos da série histórica, o $\mathrm{N}_{2} \mathrm{O}$ manteve-se em uma faixa de 52.900 e 60.500 toneladas emitidas (Figura 1).

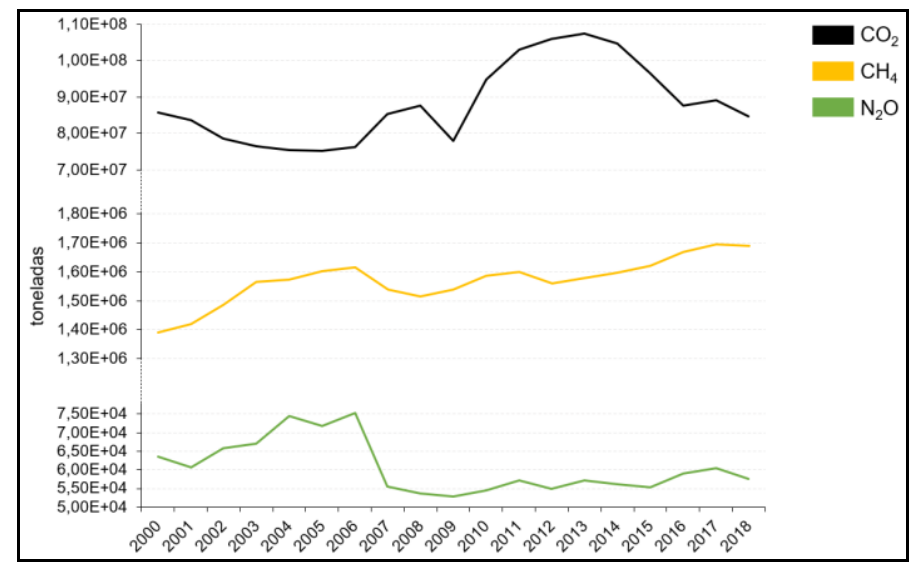

Figura 1 - Série histórica (2000-2018) dos principais GEE emitidos no estado de São Paulo. $\mathrm{CO}_{2}, \mathrm{CH}_{4}$ e $\mathrm{N}_{2} \mathrm{O}$ foram representados nas cores preto, laranja e verde, respectivamente.

Levando-se em consideração os setores econômicos analisados, o setor Energia apresentou-se como o principal emissor de GEE (Figura 2). Ao longo da série histórica, não apresentou emissões menores que 60 milhões de toneladas de $\mathrm{CO}_{2} \mathrm{eq}$, mantendo-se em uma faixa de 80 e 96 milhões de toneladas de $\mathrm{CO}_{2}$ eq emitidas.

Pode-se dizer que o setor Energia caracteriza as emissões do estado de São Paulo, sendo representado principalmente por emissões de $\mathrm{CO}_{2}$ provenientes da produção e do consumo de combustíveis fósseis, bem como da geração de eletricidade (31). Em 2018, representou 55\% das emissões totais, seguido dos setores Agropecuária e Resíduos, com contribuições de $21 \%$ e $20 \%$, respectivamente (Figura 2). 


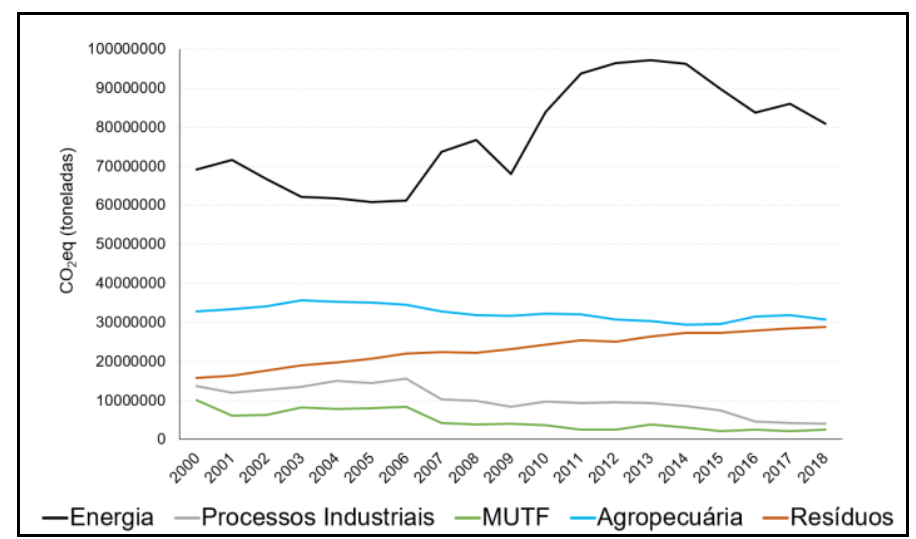

Figura 2 - Série histórica (2000-2018) das emissões totais dos principais setores econômicos do estado de São Paulo. Setores Energia, Processo Industriais, Mudanças no Uso da Terra e Florestas (MUTF), Agropecuária e Resíduos foram representados nas cores preto, cinza, verde, azul e marrom, respectivamente.

A Figura 3 apresenta as emissões de $\mathrm{CO}_{2}$ dos três principais emissores do setor Energia, a queima de combustíveis fósseis pelo setor de transportes e indústria, seguido das emissões pela produção de combustíveis. O setor de transportes destaca-se pelas elevadas emissões ao longo do período analisado, sempre superiores que 33 milhões de toneladas, mantendo-se acima de 41 milhões de toneladas de $\mathrm{CO}_{2}$ emitidas desde 2011. Assim, o setor de transportes configura-se como o maior emissor de GEE do estado de São Paulo (31).

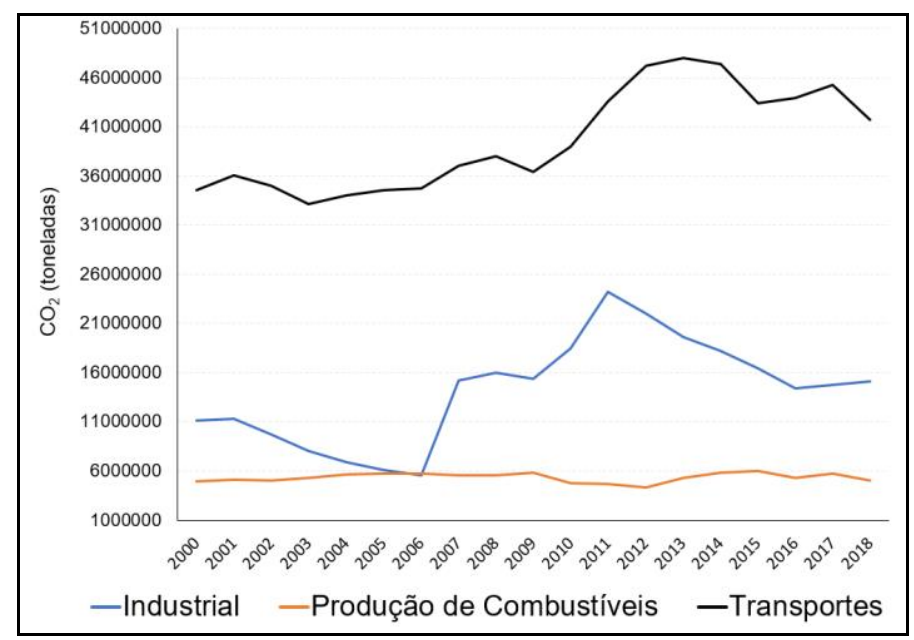

Figura 3 - Série histórica (2000-2018) das principais subdivisões do setor Energia do estado de São Paulo. Transportes, Industrial e Produção de Combustíveis foram representados pelas cores preto, azul e laranja respectivamente.

Corroborando os elevados índices de emissão de $\mathrm{CO}_{2}$ demonstrados no setor de transportes, observamos que o período analisado apresentou uma média de consumo de diesel significativamente maior que as médias de consumo de etanol e gasolina $\mathrm{C}$ (Figura 4A). Além disso, comparando as médias emissões de $\mathrm{CO}_{2}$ destes combustíveis, entre 2000 e 2018, o diesel também se destaca por apresentar média de emissão significativamente maior que as de etanol e gasolina C (Figura 4A). Não houve diferença significativa entre as médias de emissão de gasolina C (674425 \pm 14827 
toneladas) e etanol (280455 \pm 27753 toneladas), tal resultado pode ser justificado pelo elevado teor de etanol na composição da gasolina C (em torno de 27\%) (32).

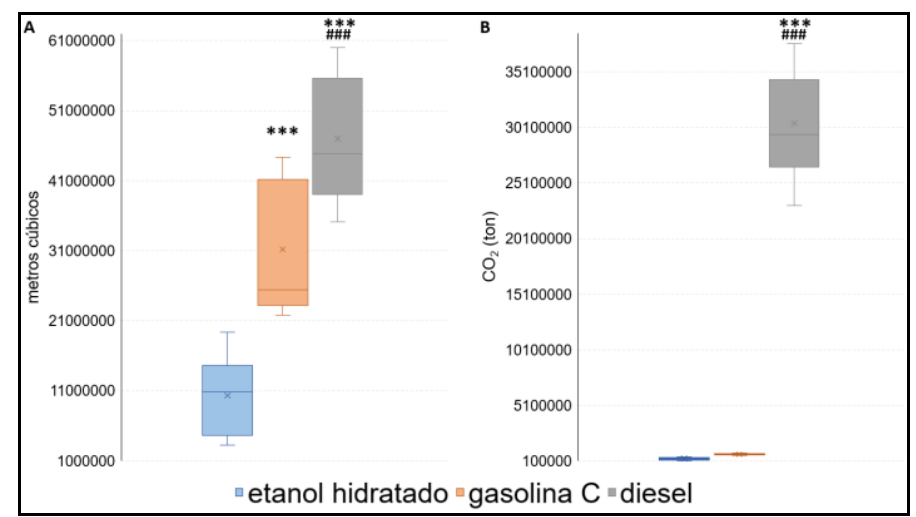

Figura 4 - Médias de consumo (A) e médias de emissão de $\mathrm{CO}_{2}(\mathbf{B})$ de diesel, gasolina C e etanol, entre 2000 e 2018. Análise realizada pelo método de comparação múltipla ANOVA, seguido do pós-teste de Bonferroni. *representa diferença em relação ao etanol; ${ }^{\#}$, em relação à gasolina $\mathrm{C}$. *** $\mathrm{e}^{\# \# \#}$ apresentam nível de significância igual de $p$ $<0,001$.

O sistema modal rodoviário brasileiro, em crescente expansão, explica a notória e indubitável participação do diesel no consumo total de combustíveis do estado de São Paulo, nos elevados índices de emissões GEE e de poluentes atmosféricos, tais como $\mathrm{NO}_{\mathrm{x}}$ e MP. Portanto, o setor de transportes desempenha papel fundamental na economia, mas também no aumento de impactos deletérios à saúde humana, relacionados à degradação da qualidade do ar (38-40).

$\mathrm{Na}$ Figura 5, podemos observar a evolução das emissões totais de $\mathrm{CO}_{2}$ e do setor de transportes, em conjunto com a evolução da mortalidade por causas respiratórias, cardiovasculares e cardiorrespiratórias (soma das duas primeiras). O período analisado foi marcado por um aumento de $38 \%, 17 \%$ e $24 \%$ destas taxas de mortalidade, respectivamente.

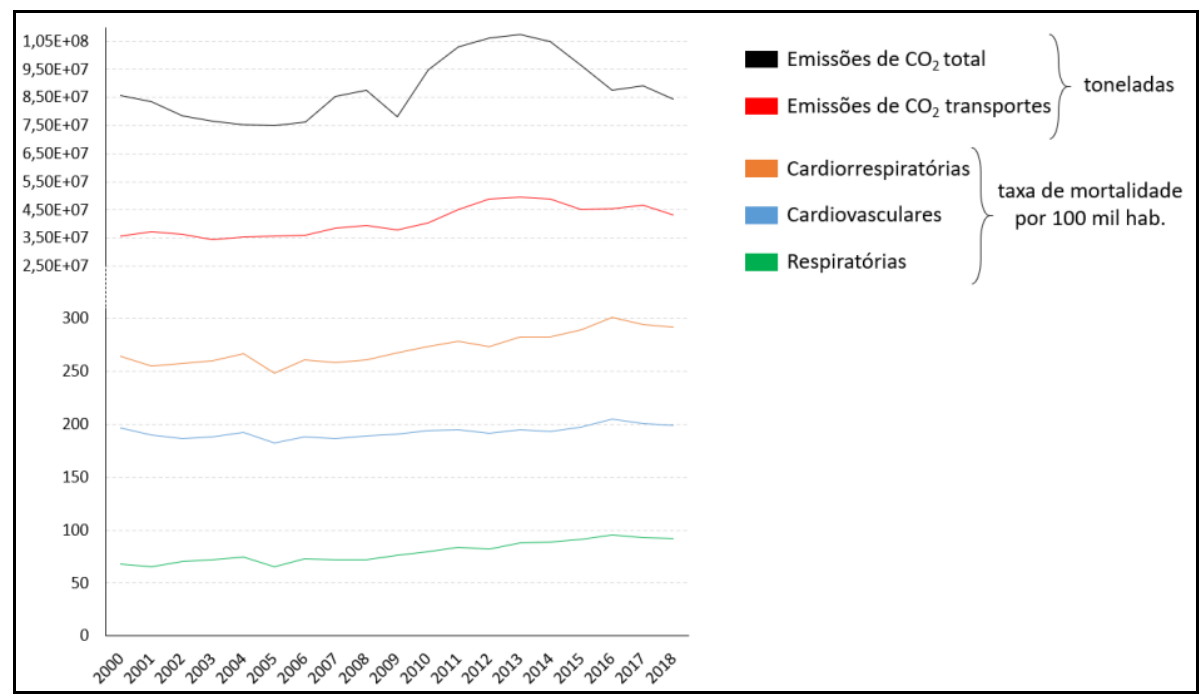

Figura 5 - Serie temporal das emissões de $\mathrm{CO}_{2}$ (totais, em preto; e setor de transportes, em vermelho) e das taxas de mortalidade por causas cardiorrespiratórias (laranja), cardiovasculares (azul) e respiratórias (verde) do estado de São Paulo (por $100 \mathrm{mil}$ habitantes), no período de 2000 a 2018. 
Na Tabela 1, podemos observar moderada correlação positiva e significativa entre as emissões totais e mortalidade por causas respiratórias $(p<0,01)$ e cardiorrespiratórias $(p$ $<0,05)$, e não significativa para doenças cardiovasculares. Considerando o setor de transportes, observa-se forte correlação positiva e significativa entre as emissões e mortalidade por causas cardiovasculares $(p<0,01)$, respiratórias $(p<0,001) \mathrm{e}$ cardiorrespiratórias $(p<0,001)$.

Tabela 1 - Análise de Correlação de Pearson para as variáveis $\mathrm{CO}_{2}$ (total e setor de transportes) e taxas de mortalidade taxas de mortalidade por causas cardiorrespiratórias do estado de São Paulo (por 100 mil habitantes), no período de 2000 a 2018.

\begin{tabular}{lll}
\hline & $\mathrm{CO}_{2}$ total & $\mathrm{CO}_{2}$ transportes \\
\hline Cardiovasculares & 0,40 & $0,60^{* *}$ \\
Respiratórias & $0,58^{* *}$ & $0,84^{* * *}$ \\
Cardiorresp. & $0,53^{*}$ & $0,78^{* * *}$ \\
\hline \multicolumn{2}{c}{$*$ para $p<0,05, * *$ para $p<0,01 \mathrm{e}^{* * *}$ para $p<0,001$}
\end{tabular}

A forte correlação positiva observada na Tabela 2 é um importante indicador de que as emissões atmosféricas provenientes do setor de transportes estão contribuindo com a manutenção dos índices elevados de óbitos e aumento das taxas de mortalidade por causas cardiorrespiratórias no estado de São Paulo. Neste contexto, é importante evidenciar que as emissões de $\mathrm{CO}_{2}$ provenientes do consumo de combustíveis fósseis, como o diesel, são acompanhadas de diversos poluentes atmosféricos, especialmente MP e $\mathrm{NO}_{\mathrm{x}}$, que estão diretamente relacionados à impactos negativos à saúde humana, uma vez que aumentam o risco de inflamação e infecção do sistema respiratório e dos agravos crônicos que provocam efeitos cardiovasculares $(18,19,41,42)$.

O estado de São Paulo apresenta milhares de quilômetros de rodovias, com fluxo intenso de veículos pesados movidos a diesel; grandes centros urbanos, com tráfico elevado de carros, motocicletas e ônibus; e apenas 54 estações fixas de monitoramento de MP em todo seu território, com diversos pontos que indicam concentrações do poluente acima das médias anuais recomendadas pela Organização Mundial da Saúde (OMS). Em 2019, o MP 10 (diâmetro aerodinâmico igual ou menor a $10 \mu \mathrm{m}$ ) apresentou concentrações anuais acima do recomendado em $92 \%$ das estações, padrão que se repete há alguns anos $(30,43,44)$.

Em termos de saúde pública, a forte dependência pela utilização de transportes movidos a derivados de petróleo e a suscetibilidade da população à exposição de poluentes associados se reflete com o aumento das hospitalizações e óbitos por causas cardiovasculares, como insuficiência cardíaca, arritmia cardíaca, doença arterial coronariana e trombose venosa; e por causas respiratórias, tais como alergias, infecções do trato respiratório, doença pulmonar obstrutiva crônica e cânceres $(18,19,41,42,45)$.

Essa dependência excessiva foi muito evidenciada, em 2018, quando ocorreu a greve dos caminhoneiros. Além do caos econômico causado e relatado em várias cidades brasileiras, um estudo recentemente publicado estimou que se os níveis reduzidos de poluentes atmosféricos observados durante a greve, provenientes de três grandes rodovias do estado de São Paulo, se mantivessem por um período de um ano, resultaria na prevenção de até 227 mortes prematuras, o que representaria uma economia de 579 milhões de dólares para o setor de saúde do estado (39).

Neste contexto, os achados aqui apresentados, mas especificamente, as emissões de 
$\mathrm{CO}_{2}$ do setor de transportes sempre superiores a 33 milhões de toneladas ao longo do período analisado, em conjunto com elevado consumo médio de diesel e com a forte correlação positiva e significativa entre as emissões e mortalidade por causas cardiovasculares e respiratórias trazem evidências importantes da contribuição do setor de transportes do estado de São Paulo na degradação da qualidade ambiental, bem como dos impactos adversos à saúde humana associados.

Além disso, nossos resultados, que são intrínsecos aos elevados níveis de poluição atmosférica estaduais, contribuem com a importante discussão sobre a urgente necessidade de revisão dos padrões de qualidade do ar pelos órgãos competentes e formulação de políticas de redução de emissões veiculares mais eficientes.

\section{Considerações finais e recomendações}

O presente estudo apresentou um panorama geral das emissões dos gases de efeito estufa do estado de São Paulo. O setor de transportes mostrou-se o principal emissor de $\mathrm{CO}_{2}$, com média elevada de consumo de diesel, refletindo na forte correlação positiva e significativa entre as emissões e mortalidade por causas cardiovasculares e respiratórias.

A análise da emissão de gases de efeito estufa e sua correlação com a mortalidade por doenças cardiorrespiratórias no Estado de São Paulo se constituem em um alerta para o direcionamento de ações com foco na mitigação desses impactos. Dessa forma, ações de mitigação da poluição do ar e a redução da dependência do consumo de combustível fóssil deveriam ser metas priorizadas na gestão ambiental pública. Diante disso, destacamos as seguintes recomendações: (1) promoção de práticas sustentáveis relacionadas à poluição do ar e emissões de $\mathrm{CO}_{2}$, tais como apoiar investimentos em transporte mais limpos, promover mobilidade urbana inteligente e inclusiva e investir em soluções multimodais para o transporte de cargas; (2) revisão dos limites de qualidade do ar estaduais pelos órgãos competentes, objetivando cumprir com os padrões determinados pela OMS; (3) formulação de políticas de redução de emissões veiculares mais eficientes, a fim de minimizar os impactos negativos à saúde humana, relacionados à exposição de poluentes atmosféricos; (4) ampliação da rede de monitoramento de qualidade do ar do estado de São Paulo, visto que muitos municípios não são contemplados com esse serviço (5) apoio e aumento de recursos para pesquisas científicas, uma vez que contribuem de forma substancial para o combate e a adaptação às mudanças climáticas.

Como uma ótima oportunidade de prática destas recomendações, a retomada da economia pós-pandemia da COVID-19 apresentará desafios quanto ao atual modelo de produção, consumo e transportes, os quais têm se mostrado insustentáveis. Retomar a economia em bases mais sustentáveis, considerando a urgência climática, é premente, configurando-se em um movimento global com ramificações no Brasil, em especial no estado de São Paulo, que concentra importantes centros urbanos. As diretrizes para uma retomada verde têm sido a necessidade de descarbonização da economia, de cidades mais sustentáveis e inteligentes e de um modo de vida baseado no consumo consciente e racional, atendendo aos ODS (Objetivos do Desenvolvimento Sustentável da ONU) e aos parâmetros ESG (Environmental, Social Governance ou, em português, Meio Ambiente, Social e Governança), os quais são considerados pelo mercado investidor.

\section{Agradecimentos}

Este estudo foi financiado pelas seguintes instituições: Fundação de Amparo à Pesquisa do Estado de São Paulo (FAPESP - 2018/26193-3), Programa Institucional de 
Bolsas de Iniciação Científica (PIBIC - no 146169/2020-4) e Coordenação de Aperfeiçoamento de Pessoal de Nível Superior (CAPES - nº 1808529).

\section{Conflito de interesses}

Os autores declaram que não existem conflitos de interesses.

\section{Referências}

1. Whitmee S, Haines A, Beyrer C, Boltz F, Capon AG, de Souza Dias BF, et al. Safeguarding human health in the Anthropocene epoch: report of The Rockefeller Foundation-Lancet Commission on planetary health. Lancet 2015;386(10007): 1973-2028.

2. Sánchez García JL, Díez Sanz JM. Climate change, ethics and sustainability: An innovative approach. J Innov Knowl 2018; 3(2): 70-5.

3. Debone D, Abe KC, Miraglia SGEK. Impact Assessment of Greenhouse Gases Emissions and Air Pollutants by Trucks on the Mortality Rate of Cardiorespiratory Diseases in São Paulo State. In In Health Impact Assessment (HIA) Case studies - Series 1. UNIFESP - SEAD; 2019. p. 211-32.

4. Espíndola IB, Ribeiro WC. Cidades e mudanças climáticas: desafios para os planos diretores municipais brasileiros. Cad Metrópole 2020; 22(48): 365-96.

5. Deng S-Z, Jalaludin BB, Antó JM, Hess JJ, Huang C-R. Climate change, air pollution, and allergic respiratory diseases: a call to action for health professionals. Chin Med J (Engl) 2020; 133(13): $1552-60$.

6. Galati EAB, Camara TN de L, Natal D, Chiaravalloti-Neto F. Mudanças climáticas e saúde urbana. Rev USP 2015; (107):79.

7. Evelyn J. Fumigugium: Or, The Inconveniencie of the Aer and Smoak of London. Together with Some Remedies Humbly Proposed. W. Godbid, editor. London; 1661. 26 p.

8. Anderson TR, Hawkins E, Jones PD. $\mathrm{CO} 2$, the greenhouse effect and global warming: from the pioneering work of Arrhenius and Callendar to today's Earth System Models. Endeavour 2016; 40(3): 178-87.

9. de Azevedo TR, Costa Junior C, Brandão Junior A, Cremer M dos S, Piatto M, Tsai DS, et al. SEEG initiative estimates of Brazilian greenhouse gas emissions from 1970 to 2015. Sci Data. 2018; 5: 180045 .

10. Beerling DJ, Leake JR, Long SP, Scholes JD, Ton J, Nelson PN, et al. Farming with crops and rocks to address global climate, food and soil security. Nat Plants 2018; 4(3): 138-47.

11. Kibria A, Akhundjanov SB, Oladi R. Fossil fuel share in the energy mix and economic growth. Int Rev Econ Financ 2019; 59: 253-64.

12. NOAA. Earth System Research Laboratory Global Monitoring Division. [Internet]. 2020. Available from: https://www.esrl.noaa.gov/gmd/dv/

13. IPCC Working Group III. Change 2014: Mitigation of Climate Change. Contribution of Working Group III to the Fifth Assessment Report of the Intergovernmental Panel on Climate Change. 2014;

14. Nori-Sarma A, Anderson GB, Rajiva A, ShahAzhar G, Gupta P, Pednekar MS, et al. The impact of heat waves on mortality in Northwest India. Environ Res 2019; 176: 108546.

15. Coumou D, Rahmstorf S. A decade of weather extremes. Nat Clim Chang 2012; 2(7): 491-6.

16. Guo Y, Gasparrini A, Armstrong BG, Tawatsupa B, Tobias A, Lavigne E, et al. Heat Wave and Mortality: A Multicountry, Multicommunity Study. Environ Health Perspect 2017; 125(8).

17. Miraglia SGEK, Gouveia N. Custos da poluição atmosférica nas regiões metropolitanas brasileiras. Cien Saude Colet 2014; 19(10): 4141-7.

18. Mannucci PM, Harari S, Martinelli I, Franchini M. Effects on health of air pollution: a narrative review. Intern Emerg Med 2015; 10(6): 657-62.

19. Gharibvand L, Shavlik D, Ghamsary M, Beeson WL, Soret S, Knutsen R, et al. The Association between Ambient Fine Particulate Air Pollution and Lung Cancer Incidence: Results from the AHSMOG-2 Study. Environ Health Perspect 2016; 125(3). 
20. Chen H, Kwong JC, Copes R, Tu K, Villeneuve PJ, van Donkelaar A, et al. Living near major roads and the incidence of dementia, Parkinson's disease, and multiple sclerosis: a population-based cohort study. Lancet 2017; 389(10070): 718-26.

21. Ambasta A, Buonocore JJ. Carbon pricing: a win-win environmental and public health policy. Can J Public Heal 2018; 109(5-6): 779-781.

22. Wang W, Saari RK, Bachmann C, Mukherjee U. Estimating transboundary economic damages from climate change and air pollution for subnational incentives for green on-road freight. Transp Res Part D Transp Environ 2020; 82: 102325.

23. Liu S, Xing J, Wang S, Ding D, Chen L, Hao J. Revealing the impacts of transboundary pollution on PM2.5-related deaths in China. Environ Int 2020; 134: 105323.

24. Debone D, da Costa M V., Miraglia SGEK. 90 Days of COVID-19 Social Distancing and Its Impacts on Air Quality and Health in Sao Paulo, Brazil. Sustainability 2020; 12(18): 7440.

25. Taconet N, Méjean A, Guivarch C. Influence of climate change impacts and mitigation costs on inequality between countries. Clim Change 2020; 1-20.

26. Hasegawa T, Fujimori S, Havlík P, Valin H, Bodirsky BL, Doelman JC, et al. Risk of increased food insecurity under stringent global climate change mitigation policy. Nat Clim Chang 2018; 8(8): 699703.

27. IBGE. Brazilian Institute of Statistics and Geography. Estimativa da População - Diretoria de Pesquisas, Coordenação de População e Indicadores Sociais. [Internet]. 2019 [cited 2020 Sep 18]. Available from: https://www.ibge.gov.br/cidades-e-estados.html

28. DENATRAN. Frota de Veículos - 2019 [Internet]. Ministério da Infraestrutura. Departamento Nacional de Trânsito. 2019 [cited 2020 Oct 30]. Available from: https://www.gov.br/infraestrutura/pt-br/assuntos/transito/conteudo-denatran/frota-de-veiculos-2019

29. CETESB. QUALAR. Sistema de Informações da Qualidade do Ar. [Internet]. 2020 [cited 2020 Sep 18]. Available from: https://qualar.cetesb.sp.gov.br/qualar/home.do

30. DER. Volume diário médio das rodovias - VDM. Departamento de Estradas de Rodagem. [Internet]. 2019 [cited $2020 \quad$ Sep 18]. Available http://www.der.sp.gov.br/WebSite/MalhaRodoviaria/VolumeDiario.aspx

31. SEEG. ANÁLISE DAS EMISSÕES BRASILEIRAS DE GASES DE EFEITO ESTUFA E SUAS IMPLICAÇÕES PARA AS METAS DO BRASIL. São Paulo, Brazil. Observatório do Clima. http://seeg.eco.br/; 2019.

32. ANP. Dados Estatísticos [Internet]. Agência Nacional do Petróleo, Gás Natural e Biocombustíveis. 2020 [cited 2020 Sep 1]. Available from: http://www.anp.gov.br/

33. DATASUS. Brazilian Health System Database (TABNET). [Internet]. 2020 [cited 2020 Sep 18]. Available from: http://datasus.saude.gov.br/informacoes-de-saude/tabnet

34. SEADE. State Data Analysis System. [Internet]. 2019 [cited 2019 Sep 18]. Available from: https://www.seade.gov.br/institucional/

35. Pereira TF, Muniz CC, Junior ESO. Emissões de CO2 em Mato Grosso por veículos automotores. Rev Ibero-Americana Ciências Ambient 2020; 11(3): 229-41.

36. Barbosa Filho F de H. A crise econômica de 2014/2017. Estud Avançados 2017; 31(89): 51-60.

37. Oliveira EC de, Pereira RS. Projeto Etanol Verde: O Protocolo Agroambiental e o compromisso com o meio ambiente no setor sucroenergético da microrregião de Assis (SP). XVI SEMEADSeminários em Adm São Paulo Anais. São Paulo-SP. 2013;16.

38. Andrade M de F, Kumar P, de Freitas ED, Ynoue RY, Martins J, Martins LD, et al. Air quality in the megacity of São Paulo: Evolution over the last 30 years and future perspectives. Atmos Environ 2017; 159: 66-82.

39. Debone D, Leirião LFL, Miraglia SGEK. Air quality and health impact assessment of a truckers' strike in Sao Paulo state, Brazil: A case study. Urban Clim. 2020;34:100687.

40. CETESB. EMISSÕES VEICULARES NO ESTADO DE SÃO PAULO [Internet]. São Paulo.; 2019 [cited 2020 Sep 1]. Available from: https://cetesb.sp.gov.br/ar/publicacoes-relatorios/

41. Zhu F, Ding R, Lei R, Cheng H, Liu J, Shen C, et al. The short-term effects of air pollution on respiratory diseases and lung cancer mortality in Hefei: A time-series analysis. Respir Med. 2019 Jan;146:57-65. 
42. Grande G, Ljungman PLS, Eneroth K, Bellander T, Rizzuto D. Association Between Cardiovascular Disease and Long-term Exposure to Air Pollution With the Risk of Dementia. JAMA Neurol 2020; 77(7): 801.

43. CETESB. QUALIDADE DO AR NO ESTADO DE SÃO PAULO. São Paulo, Brasil; 2019.

44. Corá B, Leirião LFL, Miraglia SGEK. IMPACTO DA POLUIÇÃO DO AR NA SAÚDE PÚBLICA EM MUNICÍPIOS COM ELEVADA INDUSTRIALIZAÇÃO NO ESTADO DE SÃO PAULO. Rev Bras Ciências Ambient 2020 Jul 1;1-12.

45. Miller MR, Newby DE. Air pollution and cardiovascular disease: car sick. Cardiovasc Res 2020; 116(2): 279-94. 